\title{
STAR 3 Randomized Controlled Trial to Compare Sensor-Augmented Insulin Pump Therapy with Multiple Daily Injections in the Treatment of Type 1 Diabetes: Research Design, Methods, and Baseline Characteristics of Enrolled Subjects
}

\author{
Stephen N. Davis, M.D., F.R.C.P., Edward S. Horton, M.D., ${ }^{2}$ Tadej Battelino, M.D., Ph.D., ${ }^{3}$ \\ Richard R. Rubin, Ph.D., C.D.E., ${ }^{4}$ Kevin A. Schulman, M.D., M.B.A., \\ and William V. Tamborlane, M.D., F.A.A.P., F.A.C.E. ${ }^{6}$
}

\begin{abstract}
Background: Sensor-augmented pump therapy (SAPT) integrates real-time continuous glucose monitoring (RTCGM) with continuous subcutaneous insulin infusion (CSII) and offers an alternative to multiple daily injections (MDI). Previous studies provide evidence that SAPT may improve clinical outcomes among people with type 1 diabetes. Sensor-Augmented Pump Therapy for A1c Reduction (STAR) 3 is a multicenter randomized controlled trial comparing the efficacy of SAPT to that of MDI in subjects with type 1 diabetes.

Methods: Subjects were randomized to either continue with MDI or transition to SAPT for 1 year. Subjects in the MDI cohort were allowed to transition to SAPT for 6 months after completion of the study. SAPT subjects who completed the study were also allowed to continue for 6 months. The primary end point was the difference between treatment groups in change in hemoglobin A1c $(\mathrm{HbA} 1 \mathrm{c})$ percentage from baseline to 1 year of treatment. Secondary end points included percentage of subjects with $\mathrm{HbA} 1 \mathrm{c} \leq 7 \%$ and without severe hypoglycemia, as well as area under the curve of time spent in normal glycemic ranges. Tertiary end points include percentage of subjects with $\mathrm{HbA} 1 \mathrm{c} \leq 7 \%$, key safety end points, user satisfaction, and responses on standardized assessments.

Results: A total of 495 subjects were enrolled, and the baseline characteristics similar between the SAPT and MDI groups. Study completion is anticipated in June 2010.

Conclusions: Results of this randomized controlled trial should help establish whether an integrated RT-CGM and CSII system benefits patients with type 1 diabetes more than MDI.

\section{Introduction}

$\mathbf{R}$ EAL-TIME CONTINUOUs glucose monitoring (RT-CGM), the most recent major advance in diabetes technology, provides users with sensor glucose levels that can be used for immediate and retrospective adjustments of treatment regimens. Over the past few years, a number of randomized, controlled clinical trials have been undertaken to evaluate the impact of these devices in the treatment of type 1 diabetes, and

several important observations have emerged. ${ }^{1-3}$ For RTCGM to be most effective, the devices need to be used frequently. Frequent use of RT-CGM is associated with a significant reduction in hemoglobin $\mathrm{A} 1 \mathrm{c}(\mathrm{HbA} 1 \mathrm{c})$ levels in patients who are not at the recommended level (i.e., HbA1c $>7.0 \%$ ) at baseline. ${ }^{2}$ RT-CGM can also assist people with $\mathrm{HbA} 1 \mathrm{c}$ levels $\leq 7.0 \%$ maintain target $\mathrm{HbA} 1 \mathrm{c}$ levels while reducing their exposure to biochemical hypoglycemia. Nevertheless, the relatively short (3-6 months) duration and
\end{abstract}

\footnotetext{
${ }^{1}$ Vanderbilt University, Nashville, Tennessee.

${ }^{2}$ Joslin Diabetes Center, Boston, Massachusetts.

${ }^{3}$ University Children's Hospital, Ljubljana, Slovenia.

${ }^{4}$ Johns Hopkins University, Baltimore, Maryland.

${ }^{5}$ Duke University, Durham, North Carolina.

${ }^{6}$ Yale University, New Haven, Connecticut.
} 


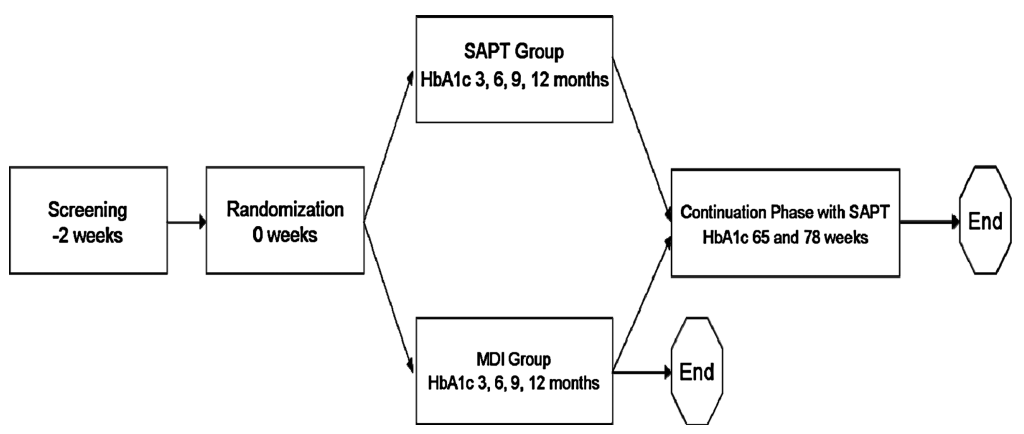

FIG. 1. Study design.

relatively small sample size of the RT-CGM studies that have been completed to date limit their generalizability. All previous studies maintained subjects on their preexisting continuous subcutaneous insulin infusion (CSII) or multiple daily injections (MDI) regimens. CSII with standard meter monitoring of glucose has been shown to improve clinical outcomes in type 1 diabetes after switching from MDI. ${ }^{4-9}$ No study to date has evaluated the effectiveness of switching directly from MDI treatment with standard meter monitoring to CSII with RT-CGM (also know as sensor-augmented pump therapy [SAPT]).

The MiniMed Paradigm ${ }^{\circledR}$ REAL-Time System (Medtronic Inc., Northridge, CA) is the only Food and Drug Administration-approved device that integrates both CSII and RT-CGM functionalities to provide SAPT in a single unit. Sensor-Augmented Pump Therapy for A1c Reduction (STAR) 3 is an industry-sponsored study that was designed to be of sufficiently large size and long duration to fill in many of the remaining gaps in evidence regarding the efficacy of RT-CGM in type 1 diabetes. Even more important, it is the first study that was specifically undertaken to compare the efficacy of SAPT with MDI treatment in subjects who were naive to CSII therapy. In this article, we present the research design and methods of the STAR 3 study, as well as the baseline characteristics of the subjects who were enrolled in the study.

\section{Research Design and Methods}

\section{Protocol development}

The initial draft of the STAR 3 study protocol was developed by the clinical research staff at the diabetes business unit of Medtronic, Inc. This protocol draft was reviewed and modified based on the recommendations of outside consultants from academic medical centers who are experts in clinical research in type 1 diabetes. The protocol was further refined and finalized following investigator meetings with study principal investigators and other clinical center staff members. A Steering Committee (members included the authors of this article and a medical director at Medtronic) was formed to oversee the conduct of the study and to guide data analysis and the preparation of articles.

A total of 30 clinical diabetes centers from the United States $(n=26)$ and Canada $(n=4)$ were involved in the study; 16 recruited only adult subjects, and nine recruited only pediatric subjects. Institutional review board approval was obtained before enrollment could begin at the site. The locations and principal investigators of each site are given in the Appendix.

\section{Study design}

STAR 3 was a randomized controlled trial with a 1-year study phase and a 6-month continuation phase (Fig. 1). To be eligible, subjects had to be diagnosed with type 1 diabetes and under the care of one of the study's primary investigators for at least 6 months before screening. In addition, they had either to be naive to CSII therapy or not used an insulin pump in the last 3 years and had baseline $\mathrm{HbA} 1 \mathrm{c}$ values $\geq 7.4 \%$ and $\leq 9.5 \%$. Subjects had to be managing their type 1 diabetes with at least three daily insulin injections (including a long-acting insulin formulation) for at least 3 months before screening. Subjects had to be performing self-monitoring of blood glucose (SMBG) an average of four times per day for at least 30 days before screening. Subjects were required to provide informed consent before enrollment.

Exclusion criteria included a diagnosis of type 2 diabetes, hypoglycemia unawareness (two or more severe hypoglycemia episodes without warning of low blood glucose [BG] levels within the previous 1 year), use of anything other than insulin to manage their type 1 diabetes in the 3 months before screening, pregnancy or intent to become pregnant, the presence of significant psychiatric or medical disorders that in the judgment of the investigator would adversely affect metabolic control or the subject's participation in the study, severe insulin resistance (i.e., insulin requirement $>2.5 \mathrm{U} / \mathrm{kg} /$ day), significant cardiovascular or renal disease, and participation in any investigational study in the 30 days before screening.

Subjects whose medical history met criteria for inclusion in the study provided written informed consent before enrollment in the study. All enrolled subjects then wore the MiniMed Guardian ${ }^{\circledR}$ REAL-Time System Clinical for 6 days to provide baseline RT-CGM data and as a further test of eligibility. Guardian System Clinical uses the same RT-CGM technology as the Paradigm SAPT System, but glucose measurements are not viewable at the time the measurement is made.

Subjects were required to complete an average of four SMBG tests over at least 5 days of blinded RT-CGM sensor wear before randomization. Subjects who successfully completed the blinded RT-CGM wear screening, met inclusion and exclusion criteria, and wished to continue in the study were randomized to either the SAPT or the MDI group. Randomization was accomplished by using an interactive voice response system. Subjects in the SAPT group used the Paradigm SAPT System and insulin aspart (NovoLog ${ }^{\circledR}$ or NovoRapid $^{\circledR}$, Novo Nordisk A/S, Copenhagen, Denmark). Subjects in the MDI group used insulin glargine (Lantus ${ }^{\circledR}$, 
Table 1. Study Summary

\begin{tabular}{|c|c|c|c|c|c|c|c|c|c|c|c|c|c|c|c|c|c|c|}
\hline & \multicolumn{17}{|c|}{ Data obtained at visit (week) } & \multirow{3}{*}{$\begin{array}{l}\text { Early } \\
\text { With- } \\
\text { drawal }\end{array}$} \\
\hline & \multicolumn{2}{|c|}{ Screening } & \multicolumn{2}{|c|}{ Enrollment } & \multicolumn{8}{|c|}{ Study phase } & \multicolumn{2}{|c|}{$\begin{array}{c}\text { Rollover } \\
\text { phase }\end{array}$} & \multicolumn{3}{|c|}{$\begin{array}{c}\text { Continuation } \\
\text { phase }\end{array}$} & \\
\hline & 1 & 2 & 1 & 2 & $2 d 2 b$ & 34 & 5 & 6 & 7 & 8 & 9 & $9 a$ & $9 b$ & $9 c$ & 10 & 11 & 12 & \\
\hline Day & -14 & -7 & 0 & 3 & 46 & 1421 & 35 & 95 & 185 & 275 & 358 & 365 & 366 & $\sim 368$ & 385 & 455 & 546 & \\
\hline \multicolumn{19}{|l|}{ Subject characteristics } \\
\hline $\begin{array}{l}\text { Informed consent/HIPAA } \\
\text { authorization }\end{array}$ & $\mathrm{x}$ & & & & & & & & & & & & & & & & & \\
\hline $\begin{array}{l}\text { Diabetes health } \\
\text { education review }\end{array}$ & $\mathrm{x}$ & $x$ & & & & & & & & & & & & & & & & \\
\hline Inclusion/exclusion criteria & & & $x$ & & & & & & & & & & & & & & & \\
\hline $\begin{array}{l}\text { Demographics/medical } \\
\text { history }\end{array}$ & & & $x$ & & & & & & & & & & & & & & & \\
\hline Height & & & $x$ & & & & & $\left.x^{*}\right)$ & $x^{*}$ & $x^{*}$ & & $x$ & & & & $x^{*}$ & $x$ & $x$ \\
\hline Weight and blood pressure & & & $x$ & & & & & $\mathrm{x}$, & $x$ & $x$ & & $x$ & & & & $x$ & $x$ & $x$ \\
\hline \multicolumn{19}{|l|}{ Clinical and safety end points } \\
\hline $\mathrm{HbA1c}$ & $x$ & & & & & & & $x$ & $x$ & $x$ & & $x$ & & & & $x$ & $x$ & $x$ \\
\hline $\begin{array}{l}\text { Medical resource utilization } \\
\text { assessment }\end{array}$ & & & & $x^{* *}$ & $x^{\dagger}$ & $x^{\dagger} x^{\dagger}$ & $x^{\dagger}$ & $x$ & $x$ & $x$ & $x^{* *}$ & $x$ & & $x^{* *}$ & $x^{* *}$ & $x$ & $x$ & $x$ \\
\hline $\begin{array}{l}\text { Serious adverse } \\
\text { event monitoring }\end{array}$ & & & $x^{* *}$ & $x$ & $x^{\dagger} x^{\dagger}$ & $x^{\dagger} x^{\dagger}$ & $x^{\dagger}$ & $x$ & $x$ & $x$ & $x$ & $x$ & $x^{* *}$ & $x^{* *}$ & $x^{* *}$ & $x$ & $x$ & $x$ \\
\hline Sensor/infusion site check & & & $\mathrm{x}$ & & $x^{\dagger}$ & $x^{\dagger} x^{\dagger}$ & $x^{\dagger}$ & $x^{\dagger}$ & $x^{\dagger}$ & $x^{\dagger}$ & & $x$ & & $x^{* *}$ & $x^{* *}$ & $x$ & $x$ & $x$ \\
\hline Device/insulin accountability & & $x$ & $x$ & $x$ & & $x^{\dagger} x^{\dagger}$ & $x^{\dagger}$ & $\mathrm{x}$, & $x$ & $x$ & $x^{* *}$ & $x$ & & $x^{* *}$ & $x^{* *}$ & $x$ & $x$ & $x$ \\
\hline \multicolumn{19}{|l|}{ Questionnaires } \\
\hline $\begin{array}{l}\text { IDSRQ adult or parent/child } \\
\text { SCREEN }\end{array}$ & & $x$ & & & & & & & & & & & & & & & & \\
\hline IDSRQ adult or parent/child & & & & & & & & $x$ & $x$ & & & $x$ & & & & $x^{* *}$ & $x$ & $x$ \\
\hline HFS-98 or HFS-P & & $\mathrm{x}$ & & & & & & $x$ & $x$ & $\mathrm{x}$ & & $x$ & & & & $x$ & $x$ & $x$ \\
\hline SF-36v2 or Peds QL & & $x$ & & & & & & & $x$ & & & $x$ & & & & & $x$ & $x$ \\
\hline Sensor Acceptance & & & & & & & & $x^{\dagger}$ & $x^{\dagger}$ & & & $x^{\dagger}$ & & & & $x^{* *}$ & $x^{* *}$ & $x$ \\
\hline CareLink Acceptance & & & & & & & & $\mathrm{x}$ & $x$ & & & $\hat{x}$ & & & & $\mathrm{x}^{* *}$ & $\mathrm{x}^{* *}$ & $x$ \\
\hline
\end{tabular}

$x$, all subjects; $x^{*}$, pediatric subjects only; $x^{* *}$, MDI subjects only; $x^{\dagger}$, SAPT subjects only; HFS, Hypoglycemia Fear Survey; IDSRQ, Insulin Delivery System Rating Questionnaire.

sanofi-aventis, Paris, France) once daily and insulin aspart with meals.

The SAPT group began using CSII at 3 days, RT-CGM at 2 weeks, and Medtronic CareLink ${ }^{\circledR}$ Therapy Management Software for Diabetes at 3 weeks. To collect comparison RT-CGM data in the control group, MDI subjects wore the blinded Guardian System Clinical for 1 week at 6 months and again at 12 months. Therapy reviews, conducted every 3 months for both groups, included notation of adverse events and vital signs, review and adjustment of insulin treatment, and administration of standardized assessments (Table 1). $\mathrm{HbA} 1 \mathrm{c}$ measurements were obtained at baseline and at 3, 6, 9, and 12 months after randomization.

At the 1-year end-of-study visit, SAPT subjects were given the opportunity to continue their present therapy, and MDI subjects were allowed to switch to SAPT therapy or complete their participation in the study. The population for the 6-month continuation phase included all subjects choosing to continue beyond 1 year. Subjects previously randomized to the MDI group began using CSII at the start of the continuation phase and began using RT-CGM and Medtronic CareLink software 3 weeks later. The HbA1c value collected at the 1-year end-ofstudy visit served as the baseline value for subjects participating in the continuation phase. During the continuation phase, HbA1c values were collected at 65 and 78 weeks.

\section{Treatment methods}

All subjects. To ensure compatibility between groups, diabetes management training was provided to all subjects, including carbohydrate counting and treatment of hypoglycemic and hyperglycemic events. They received diabetes management training at the first two visits after randomization and were administered a brief, written, standardized test to evaluate whether they retained this knowledge over several subsequent visits. To encourage consistent management across centers, investigators and subjects were provided with treatment-specific dose adjustment guidelines that were developed for the study, including worksheets, training, and reference materials about meal management, basal rates, and boluses (training and reference materials available at www.medtronicdiabetes.com). Adjustments in therapy (such as the subjects' insulin:carbohydrate ratio and suggested insulin dosing) were based on the treatment guidelines, the subject's current insulin usage, patient diaries, and SMBG data in both groups, as well as RT-CGM data in the SAPT group. This was not a treat-to-target trial; all subjects' treatment regimens were customized by investigators to achieve improvement in BG and $\mathrm{HbA1c}$ levels.

All subjects were provided with a BG meter that transmits values via radiofrequency (RF) to the Paradigm insulin pump 
or Guardian display device for RT-CGM calibration and data storage.

SAPT subjects. Subjects in this group used the Paradigm SAPT System, which integrates CSII and RT-CGM technologies and includes a Paradigm 722 insulin pump, a sensor

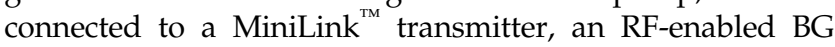
meter, and a communication interface device for uploading data to Medtronic CareLink software. The sensor calculates glucose values from the interstitial fluid and transmits them every $5 \mathrm{~min}$ to the insulin pump via the MiniLink transmitter, as previously described. Alarms and alerts allow users to know when they are experiencing a hyper- or hypoglycemic excursion. Subjects in this group were instructed to confirm their glucose value via glucose meter test and treat appropriately. Insulin usage and RT-CGM data were uploaded to Medtronic CareLink Clinical, a web-based application used only for clinical studies that records subject IDs but not names.

Device training was provided to SAPT subjects in the study phase and to MDI subjects switching to SAPT in the continuation phase. Device training began with Pump School Online, an internet-based tutorial, before initiating CSII. During the CSII initiation visit, subjects received basic insulin pump operation and programming training. SAPT subjects returned 1 week later for RT-CGM training, sensor insertion, and initiation of RT-CGM use. Subjects completed their SAPT training 1 week later, at which time they were trained in advanced insulin pump features and use of Medtronic CareLink software.

All SAPT subjects received training on how to transfer information from their pump and BG meter to Medtronic CareLink software and were encouraged to do so on a monthly basis. The insulin pump used in this study included the Bolus Wizard ${ }^{\circledR}$ calculator, which uses recently acquired data to suggest appropriate insulin boluses. SAPT users were trained on the use of the Bolus Wizard and encouraged to use this feature.

\section{Outcome measures}

Primary end point. The primary end point of this study was the difference between treatment groups in change in $\mathrm{HbA} 1 \mathrm{c}$ percentage from baseline to 1 year of treatment. All blood samples collected for $\mathrm{HbA} 1 \mathrm{c}$ tests were assessed using immunoturbidimetry at the central laboratory (Quest Diagnostics Clinical Trials, Van Nuys, CA).

Secondary end points. Secondary efficacy end points included frequency of severe hypoglycemia in each treatment group, percentage of subjects in each treatment group at months 3, 6, 9, and 12 months with an $\mathrm{HbA1}$ c value $\leq 7 \%$ and without severe hypoglycemia, and area under the curve of time spent with glucose values $>180 \mathrm{mg} / \mathrm{dL}(>9.9 \mathrm{mmol} / \mathrm{L})$ or $<70 \mathrm{mg} / \mathrm{dL}(<3.9 \mathrm{mmol} / \mathrm{L})$ in each treatment group, as calculated by RT-CGM devices. Severe hypoglycemia was defined as a hypoglycemic episode absolutely requiring assistance from another person and preferably accompanied by a confirmatory SMBG of $<50 \mathrm{mg} / \mathrm{dL}(<2.8 \mathrm{mmol} / \mathrm{L})$. If SMBG was not available, neurological recovery in response to the restoration of plasma glucose was considered sufficient evidence that the event was induced by a low plasma glucose concentration.

Tertiary efficacy end points. Tertiary end points included the percentage of subjects with $\mathrm{HbA} 1 \mathrm{c} \leq 7 \%$ at 3,6 , and 9 months of treatment, incidence of diabetic ketoacidosis, contribution of the percentage of time using RT-CGM sensors to $\mathrm{HbA1c}$ reduction, satisfaction with insulin delivery and RT-CGM systems, and results from the following standardized assessments: Hypoglycemia Fear Survey ${ }^{10}$ for adult subjects, Hypoglycemia Fear Survey-P for pediatric subjects, SF-36v2 ${ }^{\circledR}$ (Medical Outcomes Trust, Waltham, MA) for quality of life in adult subjects, ${ }^{11}$ PedsQL $^{\mathrm{TM}}$ (James W. Varni, Ph.D., College Station, TX) for quality of life in pediatric subjects, ${ }^{12}$ and Insulin Delivery System Rating Questionnaire for all subjects. ${ }^{13}$

Health economics end points. Health economics end points were derived from data obtained at baseline, 1 year, and 18 months. End points included diabetes-related hospitalizations, emergency department visits, outpatient visits, total and diabetes-related medical costs (e.g., supplies and medications), number of days lost from school or work, and indirect costs (e.g., productivity) associated with diabetes.

\section{Exploratory analysis of chronic inflammation and oxidative stress}

The CD40 ligand (CD40L) is a protein found in plasma that has been identified as an important contributor to the inflammatory process that leads to cardiovascular disease. ${ }^{14}$ Studies have linked CD40L with changes in HbA1c. ${ }^{15}$ Levels of CD40L in blood were assessed in all subjects at baseline and 1 year to determine if SAPT reduced this clinical marker of cardiovascular risk. The central laboratory for the study performed CD40L assessments using a colorimetric sandwich enzyme-linked immunosorbent assay.

\section{Sample size estimation and statistical analyses}

A sample size calculation estimated that the study required 552 adult and pediatric subjects, 276 per treatment group, plus a $10 \%$ allowance for withdrawals or nonevaluable subjects. The target sample size would provide $90 \%$ power to detect a treatment group difference as small as $0.35 \%$ in $\mathrm{HbA} 1 \mathrm{c}$ from baseline to 1 year using a two-sample $t$ test with two-sided $5 \%$ criterion significance level and an SD of $\pm 1.2 \%$. Previous studies of people with type 1 diabetes have suggested that the SD of absolute $\mathrm{HbA} 1 \mathrm{c}$ measurements (from 3 to 12 months in the studies reviewed) is between $0.9 \%$ and $1.3 \%$ in the adult ${ }^{16-18}$ and pediatric ${ }^{19}$ populations. Given the anticipated positive correlation between baseline and 1-year $\mathrm{HbA1c}$, the $\mathrm{SD}$ of the absolute $\mathrm{HbA1c}$ could be expected to be slightly lower than these estimates.

The primary study population was the intent-to-treat population, consisting of all subjects who were randomized and had at least one valid on-therapy observation for an efficacy variable. The last observation carried forward method was used for the imputation of missing values. The primary end point was the change in $\mathrm{HbA} 1 \mathrm{c}$ percentage in both treatment groups. The difference between baseline and 1-year $\mathrm{HbA1c}$ values was calculated and tested for statistical significance between treatment groups using an analysis of covariance (ANCOVA) model that included adjustment for baseline $\mathrm{HbA} 1 \mathrm{c}$ and provided a 95\% confidence interval (CI) for the treatment effect contrast. A two-sided 5\% criterion significance level was selected for the treatment effect contrast. 


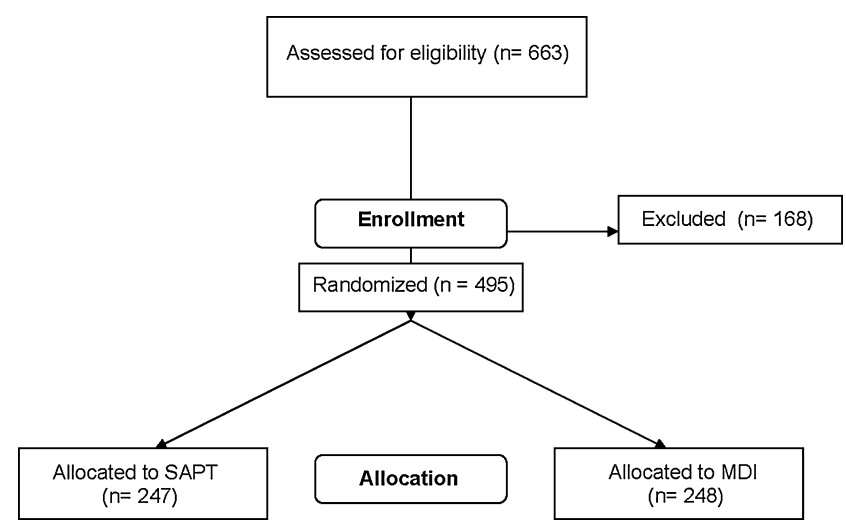

FIG. 2. CONSORT flow chart of recruitment and enrollment.

The ANCOVA model included treatment group, pooled investigative site, and age stratification (i.e., adult and pediatric subjects) as fixed effects and baseline $\mathrm{HbA1c}$ as a continuous covariate. To address the pooled investigative site factor in the primary analysis model, qualitative treatment by site interaction was evaluated by the Gail-Simon test. ${ }^{20}$ In the absence of significance for this test, the primary objective was evaluated using the overall treatment effect estimate. Mean \pm SD values and the $95 \%$ CI for percentage of change in $\mathrm{HbA} 1 \mathrm{c}$ are presented for each treatment group.

Secondary efficacy end point comparisons were performed using an ANCOVA model analogous to the primary end point. The intent-to-treat and per-protocol populations (i.e., subjects completing the study according to protocol requirements) was used for these analyses. Descriptive summaries ( $n$, mean $\pm S D$, median, range) were presented for tertiary efficacy end points without formal statistical testing.

\section{Study recruitment and baseline characteristics}

The Consolidated Standards of Reporting Trials (CONSORT) flow diagram of subject recruitment and enrollment is shown in Figure 2. Recruitment began in January 2007 and was completed in December 2008. The baseline characteristics in randomized subjects by treatment group are shown in Table 2. As can be seen, the randomization process established two groups of subjects with type 1 diabetes with similar clinical characteristics.

\section{Discussion}

Once completed, the STAR 3 study will be the largest and longest randomized clinical trial that has evaluated the efficacy of RT-CGM in children, adolescents, and adults with type 1 diabetes. In addition, it is the first trial that has been undertaken to determine whether the daunting task of implementing two advanced technologies simultaneously can be accomplished successfully. The study group includes leading diabetes treatment centers in North America that were successful in establishing two treatment groups with similar baseline clinical characteristics.

A total of 495 subjects were enrolled from January 2007 to December 2008. Most of the people who were screened but not enrolled had $\mathrm{HbA1c}$ values outside of the acceptable range or were not compliant during the blinded RT-CGM use. Data collection during the randomized controlled trial was completed in December 2009, and data analyses began in early 2010.

\section{Appendix}

\section{STAR 3 Steering Committee}

- Scott Lee, M.D. (Chair and Medical Director), Medtronic, Inc., Northridge, CA

- Tadej Battelino, M.D., Ph.D., University Children's Hospital, Ljubljana, Slovenia

- Stephen N. Davis, M.D., Vanderbilt University, Nashville, TN

- Edward S. Horton, M.D., Joslin Diabetes Center, Boston, MA

- Richard R. Rubin, Ph.D., C.D.E., Johns Hopkins University, Baltimore, MD

- Kevin A. Schulman, M.D., M.B.A., Duke University, Durham, NC

- William V. Tamborlane, M.D., F.A.A.P., F.A.C.E., Yale University, New Haven, CT

\section{STAR 3 Study Group}

The following clinical centers and primary investigators are listed in alphabetical order:

- Children's Hospital of Orange County, Orange, CA; Mark Daniels, M.D.

Table 2. BASEline Demographic Information

\begin{tabular}{lcccccc}
\hline & \multicolumn{2}{c}{ SAPT } & & \multicolumn{2}{c}{ MDI } & \\
\cline { 2 - 3 } & Adult $(\mathrm{n}=170)$ & Pediatric $(\mathrm{n}=77)$ & & Adult $(\mathrm{n}=174)$ & Pediatric $(\mathrm{n}=74)$ & All total $(\mathrm{n}=495)$ \\
\hline $\begin{array}{l}\text { Mean age [years (range)] } \\
\text { Sex [n (\%)] }\end{array}$ & $42.3(18-69)$ & $12.1(7-17)$ & & $12.6(7-17)$ & $40.0(18-66)$ & $32.4(7-69)$ \\
$\quad$ Female & $73(42.9)$ & $32(41.6)$ & & $75(43.1)$ & $34(45.9)$ & $214(43.2)$ \\
$\quad$ Male & $97(57.1)$ & $45(58.4)$ & & $99(56.9)$ & $40(54.1)$ & $281(56.8)$ \\
Race or ethnicity [n (\%)] & $9(5.3)$ & $2(2.6)$ & & $4(2.3)$ & $1(1.4)$ & $16(3.2)$ \\
$\quad$ African-American & $1(0.6)$ & $1(1.3)$ & & $2(1.1)$ & 0 & $4(0.8)$ \\
Asian & $5(2.9)$ & $2(2.6)$ & & $4(2.3)$ & $4(5.4)$ & $15(3.0)$ \\
Hispanic & 0 & $3(3.9)$ & & $2(1.1)$ & $3(4.1)$ & $8(1.6)$ \\
Other & $155(91.2)$ & $69(89.6)$ & & $162(93.1)$ & $66(89.2)$ & $452(91.3)$ \\
White & & & & &
\end{tabular}


- Children's Hospital of Philadelphia, Philadelphia, PA; Steve M. Willi, M.D.

- Children's Hospital of St. Paul, St. Paul, MN; Robert C. McEvoy, M.D.

- DeVos Children's Hospital, Grand Rapids, MI; Michael Wood, M.D.

- Diabetes Research Institute, Miami, FL; Luigi F. Meneghini, M.D., M.B.A.

- Diabetes Research Clinic, Duke University Medical Center, Durham, NC; Jennifer B. Green, M.D.

- Diabetes and Obesity Center, East Carolina University, Greenville, NC; Robert J. Tanenberg, M.D., F.A.C.P.

- Endocrine Research, Inc., Vancouver, BC, Canada; Hugh Tildesley, M.D., F.R.C.P.C.

- Endocrine Research Solutions, Inc., Roswell, GA; John C. Reed III, M.D., C.D.E.

- Endocrinology Diabetes Clinic, Madison, WI; Melissa Meredith, M.D.

- Joslin Diabetes Center, Boston, MA; Lori Laffel, M.D., M.P.H.

- Kentucky Diabetes Endocrinology Center, Lexington, KY; Lyle Myers, M.D.

- Kingston General Hospital, Kingston, ON, Canada; Robyn L. Houlden, M.D., F.R.C.P.C.

- Mayo Clinic, Rochester, MN; Yogish C. Kudva, M.D.

- Health Science Center, Memorial University of Newfoundland, St. John's, NL, Canada; Carol Joyce, M.D.

- Mid-America Diabetes Associates, PA, Wichita, KS; Richard A. Guthrie, M.D.

- Minnesota International Diabetes Center, Minneapolis, MN; Richard M. Bergenstal, M.D.

- Mountain Diabetes and Endocrine Center, PLLC, Asheville, NC; Wendy S. Lane, M.D.

- Ohio State University College of Medicine, Columbus, $\mathrm{OH}$; Kwame Osei, M.D., F.A.C.E., F.A.C.P.

- Oregon Health and Science University, Portland, OR; Andrew Ahmann, M.D.

- Rocky Mountain Diabetes and Osteoporosis Center, Idaho Falls, ID; David R. Liljenquist, M.D.

- Scripps Institute, La Jolla, CA; George Dailey, M.D.

- Toronto General Hospital, Toronto, ON, Canada; Bruce A. Perkins, M.D., M.P.H., F.R.C.P.

- Barbara Davis Center, University of Colorado Denver, Denver, CO; Robert Slover II, M.D.

- University of North Carolina School of Medicine, Chapel Hill, NC; John Buse, M.D., Ph.D., F.A.C.E.

- University of Rochester School of Medicine and Dentistry, Rochester, NY; Craig Orlowski, M.D.

- Utah Diabetes Center, Salt Lake City, UT; Carol M. Foster, M.D.

- Vanderbilt University, Nashville, TN; Stephen N. Davis, M.D., F.R.C.P.

- Washington University in St. Louis School of Medicine, St. Louis, MO; Neil H. White, M.D., C.D.E.

- Yale University, New Haven, CT; Stuart A. Weinzimer, M.D., F.A.A.P.

\section{Acknowledgments}

STAR 3 was sponsored by Medtronic, Inc. Novo Nordisk A/S supplied all insulin aspart used in the study. LifeScan, Inc., Bayer Healthcare LLC, and Becton, Dickinson and Company supplied blood glucose meters used in the study.

\section{Author Disclosure Statement}

Tim Peoples, M.A., and John B. Welsh, M.D., Ph.D., employees of Medtronic, Inc, provided writing assistance for this manuscript. Brenda Perry, M.S., R.N., a consultant to Medtronic, Inc., helped coordinate protocol development of the STAR 3 trial. The authors served as members of the STAR 3 Steering Committee. S.N.D. reports receiving consulting fees from Amylin Pharmaceuticals Inc. and sanofi-aventis US LLC. E.S.H. reports receiving consulting fees from AstraZeneca, Bristol-Myers Squibb Co., Daiichi Sankyo Co Ltd., Merck \& Co Inc., Novartis AG, F. Hoffman-La Roche Ltd., and Takeda Pharmaceuticals North America Inc. and research grants from Amylin Pharmaceuticals Inc and Eli Lilly \& Co. T.B. reports receiving consulting fees from Medtronic Inc. and Bayer AG, speaking fees from Bayer AG, Eli Lilly \& Co., Medtronic Inc., and Novo Nordisk A/S, and research grants from Abbott Diabetes Care Inc. R.R.R. reports receiving research grants from Medtronic Inc. K.A.S. reports receiving consulting fees from GlaxoSmithKline PLC, Johnson \& Johnson Inc., Medtronic Inc., Novartis AG, Novo Nordisk A/S, Pfizer Inc., and sanofi-aventis US LLC and research grants from Bristol-Myers Squibb Co., Johnson \& Johnson Inc., Medtronic Inc., Merck \& Co Inc., Novartis AG, Pfizer Inc., and sanofi-aventis US LLC. W.V.T. reports receiving consulting fees from Abbott Diabetes Care Inc., DexCom Inc., and Medtronic Inc. and honoraria from Medtronic Inc.

\section{References}

1. Bode B, Gross K, Rikalo N, Schwartz S, Wahl T, Page C, Gross T, Mastrototaro J: Alarms based on real-time sensor glucose values alert patients to hypo- and hyperglycemia: the Guardian Continuous Monitoring System. Diabetes Technol Ther 2004;6:105-113.

2. Juvenile Diabetes Research Foundation Continuous Glucose Monitoring Study Group: Continuous glucose monitoring and intensive treatment of type 1 diabetes. N Engl J Med 2008;359:1464-1476.

3. Tanenberg R, Bode B, Lane W, Levetan C, Mestman J, Harmel AP, Tobian J, Gross T, Mastrototaro J: Use of the Continuous Glucose Monitoring System to guide therapy in patients with insulin-treated diabetes: a randomized controlled trial. Mayo Clin Proc 2004;79:1521-1526.

4. Linkeschova R, Raoul M, Bott U, Berger M, Spraul M: Less severe hypoglycaemia, better metabolic control, and improved quality of life in type 1 diabetes mellitus with continuous subcutaneous insulin infusion (CSII) therapy; an observational study of 100 consecutive patients followed for a mean of 2 years. Diabet Med 2002;19:746-751.

5. McMahon SK, Airey FL, Marangou DA, McElwee KJ, Carne CL, Clarey AJ, Davis EA, Jones TW: Insulin pump therapy in children and adolescents: improvements in key parameters of diabetes management including quality of life. Diabet Med 2005;22:92-96.

6. Retnakaran R, Hochman J, DeVries JH, Hanaire-Broutin H, Heine RJ, Melki V, Zinman B: Continuous subcutaneous insulin infusion versus multiple daily injections: the impact of baseline A1c. Diabetes Care 2004;27:2590-2596.

7. Weintrob N, Benzaquen H, Galatzer A, Shalitin S, Lazar L, Fayman G, Lilos P, Dickerman Z, Phillip M: Comparison of continuous subcutaneous insulin infusion and multiple daily injection regimens in children with type 1 diabetes: a randomized open crossover trial. Pediatrics 2003;112:559-564. 
8. Wilson DM, Buckingham BA, Kunselman EL, Sullivan MM, Paguntalan HU, Gitelman SE: A two-center randomized controlled feasibility trial of insulin pump therapy in young children with diabetes. Diabetes Care 2005;28:15-19.

9. Monami M, Lamanna C, Marchionni N, Mannucci E: Continuous subcutaneous insulin infusion versus multiple daily insulin injections in type 1 diabetes: a meta-analysis. Acta Diabetol 2009 June 6 [Epub ahead of print].

10. Cox DJ, Irvine A, Gonder-Frederick L, Nowacek G, Butterfield J: Fear of hypoglycemia: quantification, validation, and utilization. Diabetes Care 1987;10:617-621.

11. Ware JE, Kosinski M, Dewey JE: How to Score Version Two of the SF-36 Health Survey. Lincoln, RI: QualityMetric, Inc., 2000.

12. Varni JW, Burwinkle TM, Jacobs JR, Gottschalk M, Kaufman F, Jones KL: The PedsQL in type 1 and type 2 diabetes: reliability and validity of the Pediatric Quality of Life Inventory Generic Core Scales and Type 1 Diabetes Module. Diabetes Care 2003;26:631-637.

13. Peyrot M, Rubin RR: Validity and reliability of an instrument for assessing health-related quality of life and treatment preferences: the Insulin Delivery System Rating Questionnaire. Diabetes Care 2005;28:53-58.

14. Granger DN, Vowinkel T, Petnehazy T: Modulation of the inflammatory response in cardiovascular disease. Hypertension 2004;43:924-931.

15. Jinchuan $Y$, Zonggui W, Jinming C, Li L, Xiantao K: Upregulation of CD40-CD40 ligand system in patients with diabetes mellitus. Clin Chim Acta 2004;339:85-90.

16. DAFNE Study Group: Training in flexible, intensive insulin management to enable dietary freedom in people with type
1 diabetes: Dose Adjustment for Normal Eating (DAFNE) randomised controlled trial. BMJ 2002;325:746.

17. Pickup J, Mattock M, Kerry S: Glycaemic control with continuous subcutaneous insulin infusion compared with intensive insulin injections in patients with type 1 diabetes: meta-analysis of randomised controlled trials. BMJ 2002; 324:705.

18. Skyler JS, Cefalu WT, Kourides IA, Landschulz WH, Balagtas CC, Cheng SL, Gelfand RA: Efficacy of inhaled human insulin in type 1 diabetes mellitus: a randomised proof-of-concept study. Lancet 2001;357:331-335.

19. Plotnick LP, Clark LM, Brancati FL, Erlinger T: Safety and effectiveness of insulin pump therapy in children and adolescents with type 1 diabetes. Diabetes Care 2003;26:11421146.

20. Gail M, Simon R: Testing for qualitative interactions between treatment effects and patient subsets. Biometrics 1985; $41 ; 367-372$.

Address correspondence to: Stephen N. Davis, M.D., F.R.C.P. Diabetes/Endocrinology Department of Medicine Vanderbilt University $7465 \mathrm{MRB} I V$ 2213 Garland Avenue Nashville, TN 37232-0475

E-mail: steve.davis@vanderbilt.edu 
